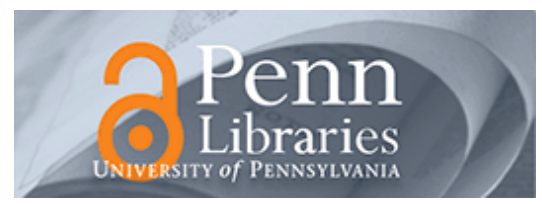

University of Pennsylvania

ScholarlyCommons

April 1989

\title{
One- and Two-Dimensional Dyadic Green's Functions in Chiral \\ Media
}

\author{
Nader Engheta \\ University of Pennsylvania, engheta@ee.upenn.edu \\ Sassan Bassiri \\ California Institute of Technology
}

Follow this and additional works at: https://repository.upenn.edu/ese_papers

\section{Recommended Citation}

Nader Engheta and Sassan Bassiri, "One- and Two-Dimensional Dyadic Green's Functions in Chiral Media", . April 1989.

Copyright 1989 IEEE. Reprinted from IEEE Transactions on Antennas and Propagation, Volume 37, Issue 4, April 1989, pages 512-515.

This material is posted here with permission of the IEEE. Such permission of the IEEE does not in any way imply IEEE endorsement of any of the University of Pennsylvania's products or services. Internal or personal use of this material is permitted. However, permission to reprint/republish this material for advertising or promotional purposes or for creating new collective works for resale or redistribution must be obtained from the IEEE by writing to pubs-permissions@ieee.org. By choosing to view this document, you agree to all provisions of the copyright laws protecting it.

This paper is posted at ScholarlyCommons. https://repository.upenn.edu/ese_papers/181

For more information, please contact repository@pobox.upenn.edu. 


\title{
One- and Two-Dimensional Dyadic Green's Functions in Chiral Media
}

\author{
Abstract \\ The one- and two-dimensional dyadic Green's functions are calculated for the one- and two-dimensional \\ electric sources in an unbounded, lossless chiral medium that is electromagnetically described \\ Comments \\ Copyright 1989 IEEE. Reprinted from IEEE Transactions on Antennas and Propagation, Volume 37, Issue \\ 4, April 1989, pages 512-515. \\ This material is posted here with permission of the IEEE. Such permission of the IEEE does not in any way \\ imply IEEE endorsement of any of the University of Pennsylvania's products or services. Internal or \\ personal use of this material is permitted. However, permission to reprint/republish this material for \\ advertising or promotional purposes or for creating new collective works for resale or redistribution must \\ be obtained from the IEEE by writing to pubs-permissions@ieee.org. By choosing to view this document, \\ you agree to all provisions of the copyright laws protecting it.
}




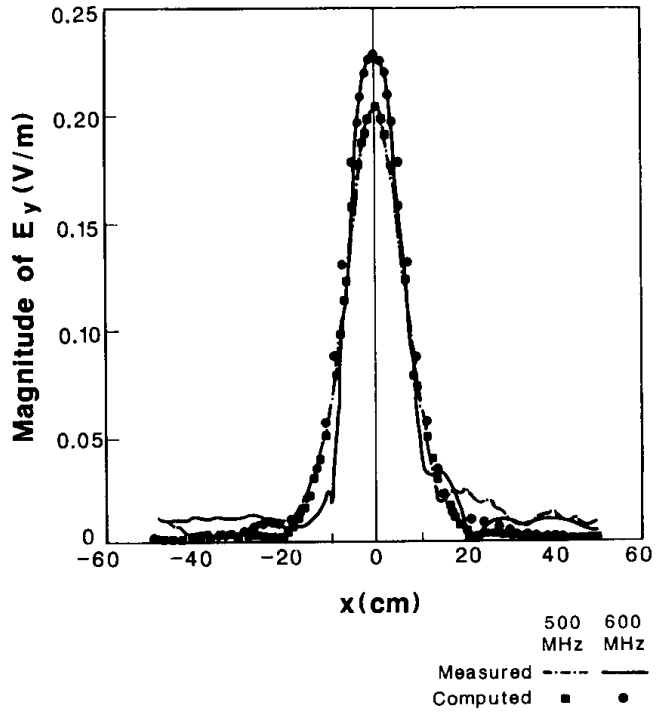

(a)

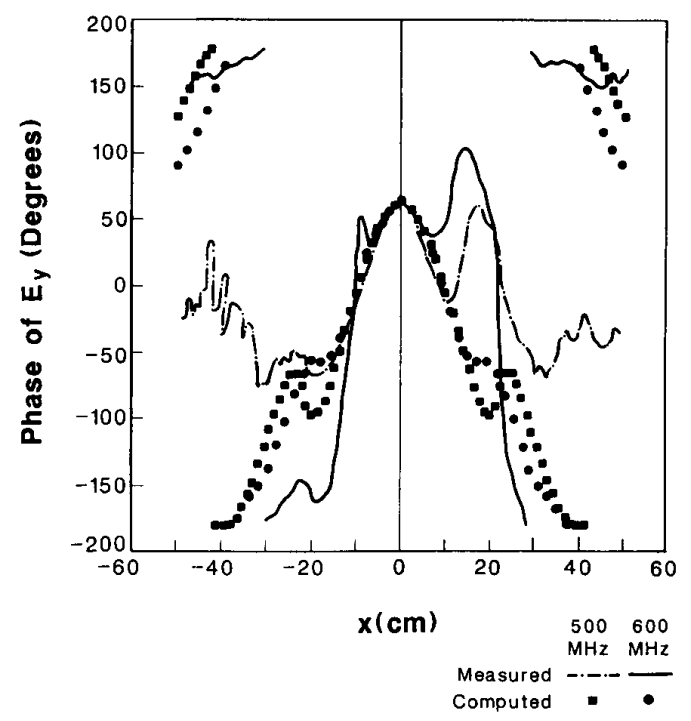

(b)

Fig. 6. (a) Measured and computed magnitudes of $E_{y}$ just above a 20 percent wet soil containing a flush-buried dielectric cylinder $(10.8 \mathrm{~cm}$ diameter and $4.78 \mathrm{~cm}$ high, $\epsilon_{p}=2.61$ ) at 500 and $600 \mathrm{MHz}$. (b) Measured and computed phases of $E_{y}$ for the flush-buried dielectric cylinder at 500 and $600 \mathrm{MHz}$.

from stray fields, so it is only noticeable in the phase measurement because of the small scattering from the objects.

\section{CONCLUSION}

An experiment has been devised to measure the magnitude and phase of the scattered fields due to flush-buried objects in order to check the associated computed results. In particular, the fields have been compared for the cases of a metal disk on the surface of the ground and more importantly for that due to a flush-buried dielectric cylinder whose dimensions are comparable to the wavelength.

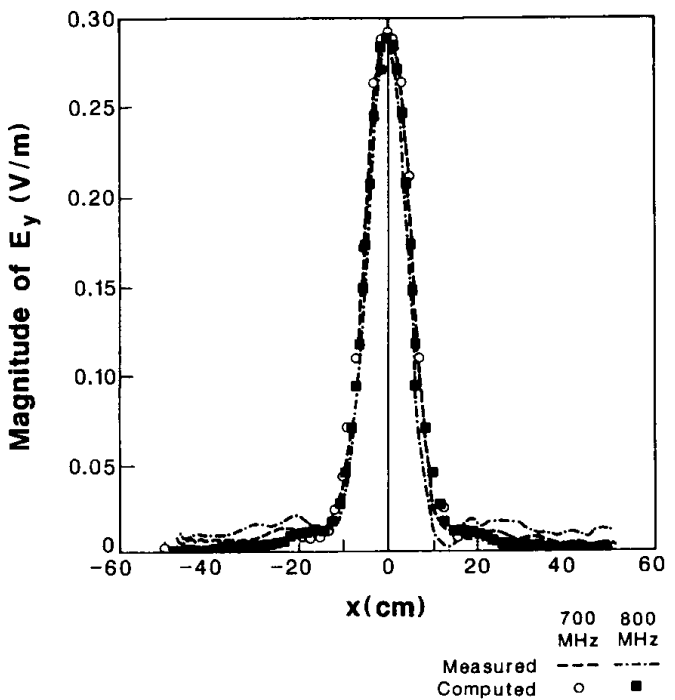

Fig. 7. Measured and computed magnitudes of $E_{y}$ for the flush-buried dielectric cylinder at 700 and $800 \mathrm{MHz}$.

\section{ACKNOWLEDGMENT}

The authors wish to thank Mr. F. D. Clapp for his assistance in the experimental portion of the investigation.

\section{REFERENCES}

[1] K. K. Mei, "Unimoment method of solving antenna and scattering problems,"' IEEE Trans. Antennas Propagat., vol. AP-22, no. 6, pp. 760-766, Nov. 1974.

[2] Y. Yee, "The measurement of the dielectric constant of earth by using the standing wave technique," M.S. thesis, Dept. EECS, University California, Berkeley, 1984.

[3] R. A. Falls and L. Mittelman, "Forecasting of the electromagnetic and thermal properties of soils by the study of their climatological environment,' Rep. 2259, MERADCOM, Ft. Belvoir, VA, September 1978.

[4] S. K. Chang and K. K. Mei, "Multipole expansion technique for electromagnetic scattering by buried objects,"' Electromagn., vol. 1, no. 1, pp. 73-89, Jan.-Mar. 1981.

[5] H. S. Chang and K. K. Mei, "Scattering of electromagnetic waves by buried and partly buried bodies of revolution," IEEE Trans. Geosci. Remote Sens., vol. GE-23, no. 4, July 1985.

\section{One- and Two-Dimensional Dyadic Green's Functions in Chiral Media}

NADER ENGHETA, MEMBER, IEEE, AND SASSAN BASSIRI

Abstract-The one- and two-dimensional dyadic Green's functions are calculated for the one- and two-dimensional electric sources in an unbounded, lossless chiral medium that is electromagnetically described

Manuscript received Mareh 18, 1988; revised July 8, 1988.

N. Engheta is with the Moore School of Electrical Engineering, University of Pennsylvania, Philadelphia, PA 19104.

S. Bassiri is with the Jet Propulsion Laboratory, California Institute of Technology, Mail Stop 238-640, Pasadena, CA 91109.

IEEE Log Number 8825326. 
by the constitutive relations $D=\epsilon E+i \gamma B$ and $H=i \gamma E+(1 / \mu) B$. The constants $\epsilon, \mu, \gamma$ are real and have values that, in general, depend on the signal frequency and the size, shape, and spatial distribution of the elements that collectively compose the medium. The results obtained in this note complement the previous work by the authors on a threedimensional dyadic Green's function in such media.

\section{INTRODUCTION}

It has been shown [2] that in the case of a chiral medium composed of lossless, short wire helices, all of the same handedness, the constitutive relations for time-harmonic fields $\left(e^{-i \omega t}\right)$ have the form

$$
\begin{gathered}
\mathbf{D}=\epsilon \mathbf{E}+i \gamma \mathbf{B} \\
\mathbf{H}=i \gamma \mathbf{E}+(1 / \mu) \mathbf{B}
\end{gathered}
$$

where $\epsilon, \mu, \gamma$ are real constants and represent the dielectric constant, permeability, and chirality admittance of the chiral medium, respectively. Moreover, it has been conjectured that (1) and (2) apply not only to chiral media composed of helices but also to any lossless, reciprocal chiral media composed of chiral objects of arbitrary shape [2]. A chiral object is a three-dimensional body that cannot be brought into congruence with its mirror image by translation and rotation. An object of this sort has the property of handedness and must be either left-handed or right-handed. Many of naturally occurring and man-made objects fall into the category of chiral objects. For instance a diverse array of sugars, amino acids, DNA and certain mollusks and winding vegetation are among the natural chiral objects while such common objects as wire helices, the Mobius strip and the irregular tetrahedron are considered the man-made chiral objects. This form of symmetry, or lack of bilateral symmetry, has been of interest to the scientific community since its discovery by Arago [3] in the early nineteenth century and subsequent experimentation by Biot [4] and Pasteur [5] in the mid-1800's. These researchers were concerned with the rotation of the plane of polarization of optical waves due to interaction with certain crystals and liquids. Since then, this phenomenon has been of interest to those in the electromagnetics' community starting with the simple but important microwave experiments of Lindman [6, 7] and Pickering [8] performed in the early and midpart of the twentieth century, respectively.

Of more recent work are papers by Bohren on the reflection of electromagnetic waves from chiral spheres and cylinders [9], [10], a paper on light reflection from chiral surfaces by Bokut and Federov [11] and the book by Kong [12] and numerous references therein regarding general bianisotropic media. Shortly thereafter was the research by Jaggard $e t a l$. on relating the interaction of electromagnetic waves with chiral stuctures and the relation of microscopic and macroscopic chiral media [2]. In the most recent past, the following papers are among those on wave propagation in chiral media: the work on transition radiation at a dieletric-chiral interface by Engheta and Mickelson [13], the reflection of waves from archiral-chiral interfaces by Silverman [14], [15] and Lakhtakia et al. [16], the electromagnetic wave propagation through a chiral slab by the authors [17], the scattering of waves from nonspherical chiral objects by Lakhtakia et al. [18], light propagation through an infinite chiral medium by Silverman and Sohn [19], the three-dimensional dyadic Green's function and dipole radiation in an unbounded, isotropic lossless chiral medium by the authors [1] and the canonical sources and duality in chiral media by Jaggard et al. [20].

In a previous paper [1], we derived from the above constitutive relations and from the time-harmonic Maxwell equations

$$
\begin{gathered}
\nabla \times \mathbf{E}=i \omega \mathbf{B} \\
\nabla \times \mathbf{H}=\mathbf{J}-i \omega \mathbf{D}
\end{gathered}
$$

the wave equation for a chiral medium. That is

$$
\nabla \times \nabla \times \mathbf{E}-\omega^{2} \mu \epsilon \mathbf{E}-2 \omega \mu \gamma \nabla \times \mathbf{E}=i \omega \mu \mathbf{J}
$$

where the source term $\mathbf{J}$ is the electric current density and where $\mathbf{E}$ is the electric vector of the radiated field. The desired solution of this wave equation was found by using the Green's function method, that is, by first constructing the dyadic Green's function $\bar{\Gamma}$ and then evaluating the expression

$$
\mathbf{E}(\mathbf{r})=i \omega \mu \int_{V^{\prime}} \overline{\mathbf{\Gamma}}\left(\mathbf{r}, \mathbf{r}^{\prime}\right) \cdot \mathbf{J}\left(\mathbf{r}^{\prime}\right) d V^{\prime}
$$

where $\overline{\mathbf{\Gamma}}$ is a function of the coordinates of the observation point $\mathbf{r}$ and of the source point $\mathbf{r}^{\prime}$, and where the integration with respect to the primed coordinates extends throughout the volume $\mathbf{V}^{\prime}$ occupied by $\mathbf{J}\left(\mathbf{r}^{\prime}\right)$. We obtained in closed form the three-dimensional dyadic Green's function for the three-dimensional electric sources.

In this note we shall follow a procedure similar to the one used in our previous work in order to find the two- and one-dimensional dyadic Green's functions for a chiral medium described by (1) and (2).

\section{The Two-Dimensional CASE}

Let us assume that the electric current density in (5) is a twodimensional current density. Hence $\mathbf{J}$ is a function of two space variables. Without loss of generality, these two space variables can be taken to be $x$ and $y$. Therefore $\mathbf{J}$ is a function of $x$ and $y$ but not $z$.

That is

$$
\mathbf{J}(\mathbf{r})=\mathbf{J}(\boldsymbol{\rho})=\mathbf{J}(x, y) .
$$

Consequently, the radiated electric field $\mathbf{E}$ in (5) and the other radiated fields $\mathbf{D}, \mathbf{B}$, and $\mathbf{H}$ are all independent of $z$.

By substituting (6) into (5) and considering (7) we see that $\bar{\Gamma}$ must satisfy the differential equation

$$
\left(\nabla^{2}+k^{2}\right) \bar{\Gamma}\left(\rho, \rho^{\prime}\right)+2 \omega \mu \gamma \nabla \times \bar{\Gamma}\left(\rho, \rho^{\prime}\right)=\left(\overline{\mathbf{u}}+\frac{1}{k^{2}} \nabla \nabla\right) \delta\left(\rho-\rho^{\prime}\right)
$$

where $\overline{\mathbf{u}}$ is the two-dimensional unit dyadic, $k^{2}=\omega^{2} \mu \epsilon$ and $\delta(\rho-$ $\left.\rho^{\prime}\right)=\delta\left(x-x^{\prime}\right) \delta\left(y-y^{\prime}\right)$ is the two-dimensional Dirac delta funciton. $\bar{\Gamma}\left(\rho, \rho^{\prime}\right)$ can be generally written as a two-dimensional Fourier integral, viz.

$$
\bar{\Gamma}\left(\boldsymbol{\rho}, \boldsymbol{\rho}^{\prime}\right)=\frac{1}{(2 \pi)^{2}} \int_{-\infty}^{+\infty} \int_{-\infty}^{\boldsymbol{\Lambda}}(\mathbf{p}) e^{i \mathbf{p} \cdot\left(\boldsymbol{\rho}-\boldsymbol{\rho}^{\prime}\right)} d^{2} p
$$

where $\mathrm{p}$ is the two-dimensional position vector and $d^{2} p$ is the surface element in the two-dimensional $p$-space, and where $\bar{\Lambda}(\mathbf{p})$ is a dyadic function of $p$. By substituting (9) into (8), the following equation can be obtained for $\bar{\Lambda}(\mathbf{p})$ :

$$
\left(k^{2}-p^{2}\right) \overline{\mathbf{\Lambda}}+2 i \omega \mu \gamma \mathbf{p} \times \overline{\mathbf{\Lambda}}=-\left(\overline{\mathbf{u}}-\frac{1}{k^{2}} \mathbf{p p}\right) .
$$

Following a procedure similar to the one used in [1], we find that

$$
\begin{aligned}
\overline{\mathbf{\Lambda}}(\mathbf{p})=\left[\left(k^{2}-p^{2}\right)^{2}+\alpha^{2} p^{2}\right]^{-1}\left\{\left(k^{2}-p^{2}\right)\left(k^{-2} \mathbf{p p}-\overline{\mathbf{u}}\right)\right. \\
\left.+\left(\alpha \mathbf{p} \times \overline{\mathbf{u}}-\alpha^{2} k^{-2} \mathbf{p p}\right)\right\}
\end{aligned}
$$

where $\alpha=2 i \omega \mu \gamma$ and $p^{2}=\mathbf{p} \cdot \mathbf{p}$. Substituting (11) into (9) we obtain 
the following expression for $\bar{\Gamma}\left(\rho, \rho^{\prime}\right)$ :

$$
\begin{aligned}
\bar{\Gamma}\left(\rho, \rho^{\prime}\right)= & -(2 \pi)^{-2}\left\{\left(\overline{\mathbf{u}}+k^{-2} \nabla \nabla\right) \int_{-\infty}^{+\infty}\left[\left(k^{2}-p^{2}\right)^{2}+\alpha^{2} p^{2}\right]^{-1}\right. \\
& \left.\cdot\left(k^{2}-p^{2}\right) e^{i \mathbf{p} \cdot\left(\rho-\rho^{\prime}\right)} d^{2} p\right\}+(2 \pi)^{-2} \\
& \cdot\left\{\alpha^{2} k^{-2} \nabla \nabla \int_{-\infty}^{+\infty}\left[\left(k^{2}-p^{2}\right)^{2}+\alpha^{2} p^{2}\right]^{-1}\right. \\
& \left.\cdot e^{i \mathbf{p} \cdot\left(\boldsymbol{\rho}-\boldsymbol{\rho}^{\prime}\right)} d^{2} p\right\}-(2 \pi)^{-2} \\
& \cdot\left\{i \alpha \nabla \times\left(\overline{\mathbf{u}} \int_{-\infty}^{+\infty}\left[\left(k^{2}-p^{2}\right)^{2}+\alpha^{2} p^{2}\right]^{-1}\right.\right. \\
& \left.\left.\cdot e^{i \mathbf{p} \cdot\left(\boldsymbol{\rho}-\boldsymbol{\rho}^{\prime}\right)} d^{2} p\right)\right\}
\end{aligned}
$$

In reducing these two-dimensional integrals to one-dimensional integrals, we follow the method used in our previous paper [1]. The one-dimensional integrals so obtained can then be evaluated by contour integration (theorem of residues). Care must be taken in choosing the path of integration in order to satisfy the physically required radiation condition [21]. Finally, the desired two-dimensional dyadic Green's function can be written as follows:

$$
\begin{aligned}
\overline{\boldsymbol{\Gamma}}\left(\boldsymbol{\rho}, \boldsymbol{\rho}^{\prime}\right)= & (i / 4)\left\{( \overline { \mathbf { u } } + k ^ { - 2 } \nabla \nabla ) \left[a H_{0}^{(1)}\left(h_{1}\left|\rho-\boldsymbol{\rho}^{\prime}\right|\right)\right.\right. \\
& \left.+b H_{0}^{(1)}\left(h_{2}\left|\rho-\rho^{\prime}\right|\right)\right]-\left(h_{2}^{2}-h_{1}^{2}\right)^{-1} \\
& \cdot\left(-i \alpha \overline{\mathbf{u}} \times \nabla+\alpha^{2} k^{-2} \nabla \nabla\right) \cdot\left[H_{0}^{(1)}\left(h_{1}\left|\rho-\boldsymbol{\rho}^{\prime}\right|\right)\right. \\
& \left.\left.-H_{0}^{(1)}\left(h_{2}\left|\rho-\rho^{\prime}\right|\right)\right]\right\}
\end{aligned}
$$

where $H_{0}^{(1)}(\cdot)$ is the zeroth order Hankel function of the first kind and where

$$
\begin{gathered}
a=\frac{\left(k^{2}-h_{1}^{2}\right)}{\left(h_{2}^{2}-h_{1}^{2}\right)} \\
b=\frac{\left(k^{2}-h_{2}^{2}\right)}{\left(h_{1}^{2}-h_{2}^{2}\right)} \\
\left\{\begin{array}{l}
h_{1} \\
h_{2}
\end{array}\right\}= \pm \omega \mu \gamma+\sqrt{k^{2}+\omega^{2} \mu^{2} \gamma^{2}} \\
k=\omega \sqrt{\mu \epsilon} \\
\eta=\sqrt{\mu / \epsilon} .
\end{gathered}
$$

The result expressed in (13) can also be obtained using an alternative method which follows. Since (8) is a result of integration of its threedimensional counterpart given in [1] with respect to the coordinates, say $z^{\prime}$ from $-\infty$ to $+\infty$, the two-dimensional dyadic Green's function $\bar{\Gamma}\left(\rho, \rho^{\prime}\right)$ can also be found through integration of the threedimensional Green's function $\bar{\Gamma}\left(\mathbf{r}, \mathbf{r}^{\prime}\right)$ reported in [1] over $z^{\prime}$ from $\infty$ to $+\infty$.

Recalling that

$$
\int_{-\infty}^{+\infty} \frac{\exp \left(i h_{j}\left|\mathbf{r}-\mathbf{r}^{\prime}\right|\right)}{4 \pi\left|\mathbf{r}-\mathbf{r}^{\prime}\right|} d z^{\prime}=\frac{i}{4} H_{0}^{(1)}\left(h_{j}\left|\boldsymbol{\rho}-\boldsymbol{\rho}^{\prime}\right|\right)
$$

which is an identity for any $h_{j}$, the integration of the three- dimensional $\bar{\Gamma}\left(\mathbf{r}, \mathbf{r}^{\prime}\right)$ over $z^{\prime}$ from $-\infty$ to $+\infty$ yields the twodimensional $\boldsymbol{\Gamma}\left(\boldsymbol{\rho}, \boldsymbol{\rho}^{\prime}\right)$ expressed in (13).

Similar to the way the three-dimensional dyadic Green's function was written by Jaggard et al. in a compact and more instructive form in terms of two eigenmodes of propagation [20], (13) can also be expressed in the following manner:

$$
\Gamma\left(\rho, \rho^{\prime}\right)=a \bar{\beta}_{1}\left(h_{1}\right) G_{1}\left(\rho, \rho^{\prime}\right)+b \bar{\beta}_{2}\left(h_{2}\right) G_{2}\left(\rho, \rho^{\prime}\right)
$$

where $\overline{\boldsymbol{\beta}}_{j}\left(h_{j}\right)$ where $j=1,2$ is an operator defined in [20] and can be written as

$$
\overline{\boldsymbol{\beta}}_{j}\left(h_{j}\right)=\left\{\overline{\mathbf{u}} \pm h_{j}^{-1} \overline{\mathbf{u}} \times \nabla+h_{j}^{-2} \nabla \nabla\right\}, \quad \text { where } j=1,2
$$

and where $G_{j}\left(\boldsymbol{\rho}, \boldsymbol{\rho}^{\prime}\right), j=1,2$ is the two-dimensional scalar Green's function in an unbounded medium and is expressed as

$$
G_{j}\left(\rho, \rho^{\prime}\right)=(i / 4) H_{0}^{(1)}\left(h_{j}\left|\rho-\rho^{\prime}\right|\right), \quad \text { where } j=1,2 .
$$

We note that

$$
h_{2} \leq k \leq h_{1}, \quad \text { for } \gamma>0
$$

and

$$
h_{1} \leq k \leq h_{2}, \quad \text { for } \gamma<0 .
$$

The eigenmode amplitudes $a$ and $b$, which were defined in [1] and [20] have positive values less than unity. The two modes, denoted by subscripts 1 and 2 , correspond to waves propagating with two different wavenumbers. It can be demonstrated that the former produce right-handed circularly polarized waves while the latter produce left circularly polarized waves in the far field.

\section{The One-Dimensional CASE}

In this case the electric current density in (5) is a one-dimensional current density. Therefore $\mathbf{J}$ is a function of space variable $x$. That is

$$
\mathbf{J}(\mathbf{r})=\mathbf{J}(\boldsymbol{x})
$$

Consequently the radiated electric field $\mathbf{E}$ in (5) and the other radiated fields $\mathbf{B}, \mathbf{H}$, and $\mathbf{D}$ are independent of $y$ and $z$.

Following a procedure similar to that in Section II, we can derive the one-dimensional dyadic Green's function for the electric sources in an unbounded reciprocal lossless chiral medium. That is

$$
\begin{aligned}
\overline{\mathbf{\Gamma}}\left(x, x^{\prime}\right)= & (i / 2)\left\{( \overline { \mathbf { u } } + k ^ { - 2 } \nabla \nabla ) \left[\left(a / h_{1}\right) \exp \left(i h_{1}\left|x-x^{\prime}\right|\right)\right.\right. \\
& \left.+\left(b / h_{2}\right) \exp \left(i h_{2}\left|x-x^{\prime}\right|\right)\right]-\left(h_{2}^{2}-h_{1}^{2}\right)^{-1} \\
& \cdot\left(-i \alpha \overline{\mathbf{u}} \times \nabla+\alpha^{2} k^{-2} \nabla \nabla\right)\left[h_{1}^{-1} \exp \left(i h_{1}\left|x-x^{\prime}\right|\right)\right. \\
& \left.\left.-h_{2}^{-1} \exp \left(i h_{2}\left|x-x^{\prime}\right|\right)\right]\right\} .
\end{aligned}
$$

The above equation can be rewritten in the following form:

$$
\bar{\Gamma}\left(x, x^{\prime}\right)=a \bar{\beta}_{1}\left(h_{1}\right) G_{1}\left(x, x^{\prime}\right)+b \bar{\beta}_{2}\left(h_{2}\right) G_{2}\left(x, x^{\prime}\right)
$$

where $\bar{\beta}_{j}\left(h_{j}\right)$ where $j=1,2$ is defined in (20) and

$$
G_{j}\left(x, x^{\prime}\right)=(i / 2)\left[\exp \left(i h_{j}\left|x-x^{\prime}\right|\right)\right] / h_{j}, \quad \text { where } j=1,2
$$

which is the one-dimensional scalar Green's function in an unbounded medium. Equation (25) can also be viewed as a result of integration of the two-dimension $\Gamma\left(\rho, \rho^{\prime}\right)$ given in (13) over $y^{\prime}$ from $-\infty$ to $+\infty$.

As can be observed from the foregoing analysis, the dyadic Green's function for electric sources in an unbounded lossless, reciprocal chiral medium can, in general, be expressed in the 
following form:

$$
\mathbf{\Gamma}\left(\mathbf{r}, \mathbf{r}^{\prime}\right)=a \bar{\beta}_{1}\left(h_{1}\right) G_{1}\left(\mathbf{r}, \mathbf{r}^{\prime}\right)+b \overline{\boldsymbol{\beta}}_{2}\left(h_{2}\right) G_{2}\left(\mathbf{r}, \mathbf{r}^{\prime}\right)
$$

where $a, b$ and $\bar{\beta}_{j}\left(h_{j}\right)$ for $j=1,2$ are defined earlier and $G_{j}\left(\mathbf{r}, \mathbf{r}^{\prime}\right)$ for $j=1,2$ depends on the dimension of the dyadic Green's function under study. That is

$$
G_{j}\left(x, x^{\prime}\right)=(i / 2)\left[\exp \left(i h_{j}\left|x-x^{\prime}\right|\right)\right] / h_{j}, \quad j=1 \text { and } 2
$$

for one-dimensional case

$G_{j}\left(\rho, \rho^{\prime}\right)=(i / 4) H_{0}^{(1)}\left(h_{j}\left|\rho-\rho^{\prime}\right|\right), \quad j=1$ and 2

for two-dimensional case

$G_{j}\left(\mathbf{r}, \mathbf{r}^{\prime}\right)=\frac{\exp \left[i h_{j}\left|\mathbf{r}-\mathbf{r}^{\prime}\right|\right]}{4 \pi\left|\mathbf{r}-\mathbf{r}^{\prime}\right|}, \quad j=1$ and 2

for three-dimensional case.

\section{SUMMARY}

In this note we have obtained the one-dimensional and twodimensional dyadic Green's functions in an unbounded, lossless, reciprocal chiral medium which is electromagnetically described by a set of symmetric constitutive relations. This work complements the authors' previous work on the three-dimensional dyadic Green's function in such media.

We have shown that in the two- and one-dimensional cases, similar to the three-dimensional case, the medium supports two eigenmodes of propagation with two different wavenumbers. One of them corresponds to the right-circularly polarized wave and the other one to the left-circularly polarized wave. The eigenmode amplitudes $a$ and $b$ are similar to those of the three-dimensional case.

\section{REFERENCES}

[1] S. Bassiri, N. Engheta, and C. H, Papas, "Dyadic Green's function and dipole radiation in chiral media," Alta Freq., vol. LV-2, 83-88, 1986.

[2] D. L. Jaggard, A. R. Mickelson, and C. H. Papas, "'On electromagnetic waves in chiral medial," Appl. Phy., vol. 18, pp. 211-216, 1979.

[3] D. F. Arago, Mem Inst., vol. 1, p 93, 1811

[4] J. B. Biot, Mem. Inst., vol. 1, p. 1, 1812, and Mem. Acad. Sci., vol. 15, p. 93,1838

[5] L. Pasteur, Ann. de Chim. et Phys., vol. 24, p. 442, 1848.

[6] K. F. Lindman, Ann. D. Phys., vol. 63, p. 621, 1920.

[7] - Ann. d. Phys., vol. 69, p. 270, 1922.

[8] W. H. Pickering, private communication, experiment performed at Caltech, 1945

[9] C. F. Bohren, "Light scattering by an optically active sphere," Chem. Phys. Lett., vol. 29, pp. 458-462, 1974.

[10] - "Scattering of electromagnetic waves by an optically active cylinder," J. Colloid Interface Sci., vol. 66, pp. 105-109, 1975. (Also C. F. Bohren and D. R. Huffman, Absorption and Scattering of Light by Small Particles. New York: Wiley, 1983.)

[11] B. V. Bokut and F. I. Federov, "Reflection and refraction of light in optically isotropic active media," Opt. Spektrosk, vol. 9, pp. 334$336,1960$.

[12] J. A. Kong, Theory of Electromagnetic Waves. New York, Wiley, 1975 .

[13] N. Engheta and A. R. Mickelson, "Transition radiation caused by a chiral plate," IEEE Trans. Antennas Propagat., vol. AP-30, pp. $1213-1216,1982$

[14] M. P. Silverman, "Reflection and refraction at the surface of a chiral medium: Comparison of gyrotropic constitutive relations invariant or noninvariant under a duality transformation," J. Opt. Soc. Am., vol. A3, pp. 830-837, 1986.

[15] _ _ "Specular light scattering from a chiral medium: Unambiguous test of gyrotropic constitutive relations," Lettere al Nuovo Cimento, vol. 43, pp. 378-382, 1985.

[16] A. Lakhtakia, V. V. Varadan, and V. K. Varadan, "A parametric study of microwave reflection," IEEE Trans. Electromagn. Compat., vol. EMC-28, pp. 90-95, 1986.

[17] S. Bassiri, C. H. Papas, and N. Engheta, "Electromagnetic wave propagation through a dielectric-chiral interface and through a chiral slab," J. Opt. Soc. Am., vol. A5, pp. 1450-1459, 1988.

[18] A. Lakhtakia, V. K. Varadan, and V. V. Varadan, "Scattering and absorption characteristics of lossy dielectric, chiral, nonspherical objects," Appl. Opt., vol. 24, pp. 4146-4154, 1985.

[19] M. P. Silverman and R. Sohn, Am. J. Phys., vol. 54, p. 69, 1986.

[20] D. L. Jaggard, X. Sun, and N. Engheta, "Canonical sources and duality in chiral media," IEEE Trans. Antennas Propagat., vol. 36 , pp. 1007-1013, 1988.

[21] A. Sommerfeld, Partial Differential Equations in Physics. New York: Academic, 1949.

\section{Wide-Band Microwave Diffraction Tomography Under Born Approximation}

\author{
TAH-HSIUNG CHU AND KEN-YU LEE
}

Abstract-Studies of the diffraction tomography of dielectric objects in forward and backward scattering using a frequency diversity technique in the microwave region are presented. Numerical results show that the image reconstructed in the backward scattering case is better than that obtained in the forward scattering case. This shows that this cost-effective technique has potential in medical and nondestructive testing applications.

\section{INTRODUCTION}

It was shown that under Born approximation and plane wave illumination, a two-dimensional object function (or dielectric tomographic image) can be reconstructed from the scattering data collected by a linear array using angular diversity techniques in forward scattering [1]. This is known as the Fourier diffraction projection theorem [2]-[4], and has been extensively applied in the area of acoustical imaging [5]-[7]. In this communication, we present studies of microwave diffraction tomography using frequency diversity techniques in forward and backward scattering, and numerical results obtained in the frequency range $(1-6) \mathrm{GHz}$. It is shown that this technique in backward scattering can appreciably reduce the number of views and has potential in medical and nondestructive applications.

\section{THEORETICAL ANALYSIS}

A weakly scattering two-dimensional object (see Fig. 1), possessing a nondispersive refractive index $n\left(x^{\prime}, y^{\prime}\right)$ in a lossless medium assumed air here, is illuminated by a monochromatic plane wave propagating in the $x$-direction. The scattered field $U_{s}\left(k_{0}, x= \pm d\right.$, $y$ ) accessed by a linear array located at $x=d$ (forward scattering) or $x=-d$ (backward scattering) satisfies the scalar Helmholtz

Manuscript received August 19, 1987; revised July 13, 1988. This work was supported by National Science Fund Grant NSC 76-0404-002-32 of the Republic of China.

The authors are with the Electrical Engineering Department, National Taiwan University, Taiwan, Republic of China.

IEEE Log Number 8825324. 